\title{
The Challenge that Abstract Concepts Pose for Simulation-based Theories
}

\author{
Hecun Liu ${ }^{1, *}$, Bolin Yang ${ }^{2}$ \\ ${ }^{1}$ School of Human Health Science, Purdue university, West lafayette, Indiana 47907, United States. \\ ${ }^{2}$ School of Mary Star of the Sea, 2500 N. Taper Ave. San Pedro, California, 90731, United Sates. \\ *Corresponding author Email: liu3166@purdue.edu by11835@marystarhigh.com
}

\begin{abstract}
According to recent studies on embodied cognition, we believe that cognitive activities of the brain are closely related to people's modality-specific area, non-modality-specific area, and people's perceptual-motor experience. To better understand how people relate abstract concepts to real experiences, we analyze the metaphorical hypothesis, the situational hypothesis, and the affective false correlation studies. We find that both the metaphor hypothesis and the situational hypothesis have some limitations. In addition, we have found that emotions contribute to the understanding of abstract concepts. So we further discuss and analyze the emotion hypothesis. Finally, we conclude that abstract concepts cannot be fully understood by relying on a single method; Instead, the interplay of metaphors, situations, and emotions helps shape people's existing perceptions. Overall, this work provides people new insights into understanding the relationship between abstract concepts and human experience. The study also sheds light on how diverse the brain is in understanding things.
\end{abstract}

Keywords: Embodied cognition, Emotion, Abstract concept

\section{INTRODUCTION}

\subsection{The challenge Embodied Theory is Facing}

Embodied view is a simulation-based theory that was first proposed by Lawrence W. Barsalou et al. and was compared to the disembodied view. After experiments and papers, researchers finally have concluded the embodied theory as: when people read motor information or verbs related to specific body parts, both our corresponding motor cortex (modality-specific area) and linguistic area (amodal area) in our brain will be activated (e.g. when people heard the verb "kick", our specific leg motor cortex will be stimulated) [1]. In other words, embodied theory supports "thinking involves perceptual-motor simulations" [2]. However, in our daily life, we not only just use concrete words such as color, verbs, and smells to describe and to represent objects and actions, but also use the abstract words to represent things where we cannot see or touch such as time, love, and justice. These are the abstract concepts, which the embodied theory cannot explain. In previous study, Willems, Simmons, Gonzalez, and etc, have given strong evidence for embodied theory where the results showed that the words related to motion, color, and olfaction activated our modality-specific area but none of these were activated for the abstract concepts[3-5]. That is to say, although we experience abstract concepts, we do not have a specific organ to sense and to perform the abstract concepts as we have organs to perform actions and the other concrete words [2]. Thus, abstract concepts posed a big challenge to embodied theory.

Metaphor theory provides new explanations to address the challenges faced by the embodied cognition theory. Metaphor theory holds that people can use simple and concrete concepts (temperature and space) to understand complex and abstract concepts (such as interpersonal relationships and psychological perception) In other words, people use mental representations to form a source field (concrete) to help people think about an abstract target field.

Research supporting metaphor theory is persuasive. In their study, Casasanto and his colleagues found an asymmetric cross-dimensional interference pattern between time and space through six experiments. Through the asymmetrically between time and space, researchers found the correlation relationship: the effect of distance on time estimation is greater than the effect of time on distance estimation [6]. In another study on direct 
movement and vocabulary learning, Casasanto and another colleague through three experiments found that the activation of a metaphor from space to valence improved learning of positive or negative words (metaphor improved learning) [7]. All of these studies provide strong evidence for metaphors.

\subsection{Metaphor theory may not be the (only) solution.}

Even though we have good evidence that metaphor theory helps people understand abstract concepts, we do not have good evidence that the metaphor is embodied, which is the limitation of metaphor theory. Many studies try to support the metaphor is embodied. People think we can understand "grasping the concept" and "kicking the habit" by creating a source domain that is represented by physically grasping and kicking. If that is the case, we should see that some areas are activated in the brain motor system. So to prove it, Aziz-Zadeh and his colleagues made the first attempt. They gave people literally scenes like kicking the ball or grasp the cup, and they found foot and hand areas were activated. Next, they tried to find out if foot and hands areas were still activated when people said "kick the bucket" or "grasp the idea". However, they found no convincing evidence that the somatosensory cortex was activated when people said "kicking the bucket" or "grasping the idea" [8]. Many subsequent studies carried out similar attempts. For example, Quadfleig thinks that if brains can discriminate above the line from below the line in visual space, can people use the same information in their brain to discriminate up/down valenced words (positive and negative emotional valence words)? However, the final results shown that the overlaps of brain regions only appeared in modality-non-specific areas [9]. Besides, many other studies did not find any good evidence of the embodied source domain.

\subsection{Potential Solutions other than Metaphor Theory}

So the limitations of metaphor theory have prompted people to look for other potential solutions. In our view, there are two other potential explanations -- situational and emotional.

\subsection{Situational Model}

Since metaphor theory can hardly function as a potential solution to the embodied theory, situational model seemed to be a plausible explanation. The situational model supports that people do not need all of the details to build a meaning-related scene to assist comprehending while people are reading abstract texts. "Situation models are mental representations of the state of affairs described in a text rather than of the text itself."
[10] For example, when people read "a pen is on a piece of paper", people do not need the information of the design of the pen, the size of the paper, or etc, to form the scene mentally. To the scene, people represent situational model based on our personal experiences. In other words, the awareness and memory such as different people around us, different environments around us, and objects relevant to our goal, influenced and changed the specifics of the situational model. This theory may share some similarities with metaphor theory. Nevertheless, compared to the abstract relation between the source domain and the target domain of the metaphor theory, the representation in the brain of the situational model theory is more concrete, specific, and approximately more likely to be embodied. In any way, the situational model provided a new perspective to search solutions to the embodied theory with certainty. Moreover, numbers of research showed that the situational model focuses on mental representations that are constructed by words rather than the word itself, which intend to support the situational model is embodied. Through Barclay's experiment, people tended to recall the memory more quickly when the test phrases were matched to the encoding phrases and more slowly when the phrases were not matched. The situational model is dependent on memory and early experiences so much that the recent environments and events even when they have just happened seconds ago, can barely influence the forming and activating of situational models [11].

However, the situational model has a fatal problem in which the situational theory is actually a disembodied theory. According to Bower's research, the situational model is an emotional-related theory where people in a happy mood are able to recall happy memories more easily and vice versa. In the conclusion, Bower referred that these have matters to do with the nodes of imaginary such as evoking appraisals and autonomic patterns, and the contacts between neurons [12]. Thus they were not able to assume that situational model is embodied. Furthermore, Kintsch had given a more clearer diagram years later to explain how the theory works in linguistic areas. It introduced the general process of words producing their proposition and they being elaborated, integrated, and performed or transformed into memory [13]. Nevertheless, they all supported the disembodied theory.

\subsection{Emotional Theory}

Barsalou and Wimer-Hastings (2005) proposed that abstract concepts and lexical meanings are rooted in introspective states (psychological and emotional). So this caused us to think that in addition to metaphors and situations, perhaps some abstract concepts can also be reflected in emotional experience. 
In Kousta's experimental study of abstract words and emotions, they found that abstract concepts are different from concrete concepts. And abstract concepts seem to be more closely related to people's judgments of emotions. One can clearly state whether abstractions are positive or negative. But for concrete concepts, the connection is not so strong. Kousta (2011) believes that emotions will affect the speed of people's understanding of concepts. So researchers inferred that emotion is the most critical factor in motivating people to understand abstract things [14].

So, through the experiments mentioned above, as well as some other literature, we think maybe some abstract concepts can be embodied during the emotional experience.

\subsection{Paper Review}

To support our idea, we found a study on the relationship between emotion and semantic size. This article provides the basis for emotional interpretation.

We all know that the size of specific objects can be linked to visual experience. What about an abstract concept that doesn't exist in space-time, does it have a size? Intuitively, "trust" and "forever" feel bigger than "traces" and "humility." The same concept, such as an idea, can be big or small in different contexts. Sometimes we say, "I like big ideas," and sometimes we say, "My ideas are small." So how does the brain represent the size of an abstraction?

So thinking about the size of abstract words is the inspiration of this article. On this basis, the researchers conducted experiments to find out whether there is a correlation between emotions and understanding abstraction [15].

In this paper, the researchers first focused on the relationship between semantic size and people's recognition of concrete and abstract words. Next, the researchers looked at the relationship between semantic size of words and affective characteristics (arousal and valence). The researchers hypothesized that people would respond faster to words representing concepts of larger objects than words representing concepts of smaller objects. In addition, the researchers also hypothesized that there is a strong correlation between the semantic size of words and their affective characteristics. In addition, compared with concrete words, there was a stronger relationship between the semantic size of abstract words and emotionality. To test the hypothesis, the researchers designed experiments. In the experiment, participants were presented with half the words and half the non-words. Next, the participant had to press the appropriate response button as quickly and accurately as possible. The results show that people process semantic big words better than small words. And semantically large words have the same advantage in dealing with both concrete and abstract words. But we've known before that the exact size of an object can be correlated with what people experience in terms of vision. But abstract concepts cannot be embodied in people's visual experience. The researchers argue that we still don't know how people process abstract words and why semantically large words can provide a processing advantage over abstract concepts. So the researchers extended the size effect from concrete to abstract concepts through a series of correlation analyses and regression analyses. The effect of emotional arousal on size was also assessed, as the researchers found that emotional arousal was the only significant predictor of size. The results show that semantic size is highly correlated with the subjective rating of emotional arousal. In other words, the size of emotional intensity can help people process the size of abstract concepts. In response to these results, the researchers proposed an emotional explanation theory. The researchers believe that in addition to situations and metaphorical methods, people's emotions also influence how they interpret abstract concepts.

However, the results of this experiment only supported correlations between emotions and abstract concepts. But the study doesn't provide enough evidence to support the embodied theory of emotions. So, looking for evidence that the emotion theory is embodied, we designed our own experiment.

\section{METHOD}

\subsection{Designed Experiment}

To further support the idea that emotion theory can help people understand abstract concepts, and emotion theory can be embodied, we designed three experiments.

\subsection{Experiment 1 design}

In the first experiment, we will discover the correlation between emotion and abstract words by observing the difference in the influence of emotion on abstract words and the difference in the influence of emotion on concrete words. We will match with 10 groups (negative, positive, neutral) abstract words and 10 groups (negative, positive, neutral) concrete word. Then we will measure the response time.

\subsection{Experiment 2 design}

We will intervene people's emotional state through external intervention, such as using sad or happy events to influence people's emotional state, and then observe whether people's understanding of positive or negative abstract concepts will change.

Finally, in Experiment 2, we tried to demonstrate that emotion is embodied during the understanding of abstract concepts. Previous studies showed that the facial 
expressions of people affect the emotional expression of individuals. And facial expressions can also affect the internal physiological activities, which proved that emotions can be embodied.

As we assume that when people change their facial expressions, the related modality-specific area of the brain will be activated. We then tell participants to change their facial expressions to influence their emotions, and then let them recognize abstract words. We will use fMRI to observe which modality-specific areas are activated when the subjects change their facial experience (frowned or smiled). Then, when the participants are recognizing and understanding the abstract words, we would observe whether the corresponding modality-specific areas of the subjects' brains were also activated. If the activation areas match, this experiment could therefore provide evidence that emotional interpretation can be embodied.

\section{CONCLUSION}

We've talked about how abstract concepts can borrow metaphorically from specific experiences, and how they can relate to relevant situations. Through the analysis of previous studies, we know that metaphor and situational theory have the same limitations. Neither of these can be well demonstrated to be embodied. For a long time, people have focused on the study of metaphor and situation theory, but neglected the study of emotion theory. Through the analysis of relevant literature, we found that people's perception of abstraction can also come from emotional experience, and emotion can sometimes help people understand abstract concepts. In addition, our research shows that metaphor, situation, and emotion are not mutually exclusive.

For example, we understand our feelings, such as love and hate, through concrete experiences we have had, including the way we remember the situation and the emotions we once experienced. Therefore, the brain's learning and understanding of the abstract concept of "love" or "hate" is likely very diverse. From this example, we can find that one cannot fully understand abstract concepts by relying only on a single method; Instead, the interplay of metaphors, situations, and emotions helps shape people's existing perceptions. And people gain different experiences in growing up, and these different experiences make people have different understandings of emotions. So everyone understands the same abstract concept differently. From our point of view, this is what distinguishes people from computers and machines. To have new experiences, we humans must constantly interact with the world. Only in this way can we gain new and diverse understandings of things.

At present, researches on metaphor theory, situation theory, and emotion theory still have some limitations. Future studies need researchers to find evidence that these three methods can be embodied. Future research should also focus on further studying the relationship between these three methods. And find more experimental evidence to support the relationship between these three complementary.

\section{REFERENCES}

[1]Pulvermüller F. (2005) Brain mechanisms linking language and action. Nat Rev Neurosci 6, 576-582. https://doi.org/10.1038/nrn1706

[2]Casasanto D. (2021) Daniel Casasanto-Human Cognition: Understanding the mind in context, In: lecture 5, online, pp. 2.

[3]Willems, R., Labruna, L., D'Esposito, M., Ivry, R., \& Casasanto, D. (2011). A Functional Role for the Motor System in Language Understanding: Evidence From Theta-Burst Transcranial Magnetic Stimulation. Psychological Science, 22(7), pp.849854. Retrieved April 10, 2021, from http://www.jstor.org/stable/25835465

[4]W. Kyle Simmons, Vimal Ramjee, Michael S. Beauchamp, Ken McRae, Alex Martin, Lawrence W. Barsalou (2007) A common neural substrate for perceiving and knowing about color, Neuropsychologia, Volume 45, Issue 12, pp. 2802 2810. https://doi.org/10.1016/j.neuropsychologia.2007.05. 002

[5]Julio González, Alfonso Barros-Loscertales, Friedemann Pulvermüller, Vanessa Meseguer, Ana Sanjuán, Vicente Belloch, César Ávila (2006) Reading cinnamon activates olfactory brain regions, NeuroImage, Volume 32, Issue 2, pp. 906-912. https://doi.org/10.1016/j.neuroimage.2006.03.037

[6]Casasanto, D., Boroditsky, L. (2008). Time in the mind: Using space to think about time. Cognition, 106(2),

pp.579-593. https://doi.org/10.1016/j.cognition.2007.03.004

[7]Casasanto, D., Bruin, D. A. (2019). Metaphors we learn by: Directed motor action improves word learning. Cognition, 182, pp.177-183. https://doi.org/10.1016/j.cognition.2018.09.015

[8]Aziz-Zadeh, L., Wilson, M. S., \& Rizzolatti, G., \& Iacoboni, M. (2006). Congruent embodied representations for visually presented actions and linguistic phrases describing actions. Curr Biol, 16(18), pp.1818-1823. https://doi.org/10.1016/j.cub.2006.07.060

[9]Susanne Quadflieg, Joset A. Etzel, Valeria Gazzola, Christian Keysers, Thomas W. Schubert, Gordon D. Waiter, C. Neil Macrae (2011) Puddles, Parties, and 
Professors: Linking Word Categorization to Neural Patterns of Visuospatial Coding. J Cogn Neurosci, $23 \quad(10)$, pp.26362649. https://doi.org/10.1162/jocn.2011.21628

[10]Zwaan RA. (1999) Situation Models: The Mental Leap Into Imagined Worlds. Current Directions in Psychological Science. 8(1): pp.15-18. https://doi.org/10.1111/1467-8721.00004

[11]Elizabeth F. Loftus, John C. Palmer (1974) Reconstruction of automobile destruction: An example of the interaction between language and memory,

Journal of Verbal Learning and Verbal Behavior, Volume 13, Issue 5, pp.585-589. https://doi.org/10.1016/S0022-5371(74)80011-3

[12]Bower, G. H. (1981). Mood and memory. American Psychologist, 36(2), pp.129148. https://doi.org/10.1037/0003-066X.36.2.129

[13]Kintsch, W. (1992). A cognitive architecture for comprehension. In: H. L. Pick, Jr., P. W. van den Broek, \& D. C. Knill (Eds.), Cognition: Conceptual and methodological issues (p. 143-163). American Psychological

Association. https://doi.org/10.1037/10564-006

[14]Kousta, T. S., Vigliocco, G., \& Vinson, P. D., \& Andrews, M., \& Campo, E. D. (2011). The representation of abstract words: why emotion matters. J Exp Psychol Gen, 140(1), pp.14-34. https://doi.org/10.1037/a0021446

[15]Yao, B., Vasiljevic, M., Weick, M., Sereno, M. E., O’Donnell, P. J., \& Sereno, S. C. (2013). Semantic size of abstract concepts: It gets emotional when you can't see it. Plos one, 8(9), e75000. https://doi.org/10.1371/journal.pone.0075000 